\title{
Power Allocation Algorithm of OFDM-Based Cognitive Radio Network using Particle Swarm Optimization
}

\author{
Nilesh Bobde ${ }^{1}$, Dr. V. V. Dixit ${ }^{2}$ \\ ${ }^{1}$ M.E. student, SCOE, Pune, India \\ ${ }^{2}$ Associate Professor, SCOE, Pune, India
}

\begin{abstract}
The deficiency of wireless spectrum resources is one of the main factors constraining the further development of wireless communication. To improve resource efficiency, Cognitive Radio (CR) one of the best solution to improve. Cognitive Radio is novel concept which allow wireless systems to sense the environment, adapt and learn from previous experiences to improve quality of communication. Orthogonal frequency division multiplexing (OFDM) used for its flexible dynamic resource allocation hence used this OFDM technique in the cognitive radio. It also assures no mutual interference among the CR radio channels which are adjacent each other. A distributed algorithm is an algorithm design to run on computer hardware constructed from interconnected processor. The drawback of the distributed algorithms is more component to fail and security problem. In this paper, we maximize the system throughput and suboptimal power allocation for multiuser OFDM CR system. For demonstrating the suboptimal power allocation, we used the Particle Swarm Optimization (PSO) algorithm.
\end{abstract}

Keywords: Cognitive Radio, OFDM, Particle Swarm Optimization

\section{Introduction}

With the rapid development of wireless devices, technology and communication services, new frequency band are being used in the radio spectrum. Due to increase in the wireless device count, the radio spectrum is becoming increasingly congested. Also the increase in the new wireless devices with the development in technology has promised more and more frequency band to be utilized. This may result in the high level of interference among the frequency bands which are being operated adjacent to each other. Again, it depends on the time and place of use. Hence unlicensed spectrum bands and reserved spectrum resources are increasingly unable to satisfying the upcoming services which require spectrum management policy. Generally, the frequency bands which are licensed operated at fixed time and remaining time they are free. These free or unused bands of the spectrum cannot be used by conventional wireless system because these are licensed and can be used by only respective owners of the band. So, to use these bands which are which are unused by licensed user during certain time [1].

For that, we used the Cognitive Radio (CR) as a device which automatically sense the unused frequency band and also automatically change parameters. Cognitive radio is an intelligent radio that can be programmed and configured dynamically. Cognitive radio also known as smart radio with an intelligent technology which can learns its radio environments and changes its transmission parameters. It can adapt itself to decide the future actions dynamically to improve the communication quality and meet the overall requirements of the users. The main feature of CR system is that it is autonomous and software controlled. It can change its characteristics dynamically without the involvement of other users. This involves the sensing of the free spectrum i.e. spectrum hole and then deciding radio resources such as symbol rate, power, bandwidth, number of subcarriers etc. to the group of secondary (or CR) users based on the behavior of users to whom the frequency band is licensed (primary users). All of these processes are controlled by software and are fully dynamic in nature [2].

The spectrum sensing includes detection of unused spectrum from wireless band which results in minimum interference with other users. The free frequency bands are known as spectrum holes. Various techniques which are used to detect such as transmitter detection, Matched filter Detection, Energy Detection, etc. After the proper frequency has been sensed, the problem of spectrum management arrives. It requires allocation of various parameters on which data transmission takes place. If the operating channel meets with interference level above threshold, then frequency of operation needs to be changed in a smooch manner, not derange the existing data exchange [3].

OFDM stands for Orthogonal Frequency Division Multiplexing. It is multicarrier modulation technique in which data is split up into chunks and every chunk is modulated using closely spaced orthogonal subcarrier. OFDM is used for allocated unused spectrum flexibility. The orthogonal subcarriers have property that they do not have any mutual interference between them and this is very helpful for high bit rate data communication. One of the serious problems of high data rate transmission is time dispersion of pluses resulting in Inter-Symbol Interference (ISI). In OFDM, data is split into several low rate data chunks and are modulated in overlapping orthogonal subcarriers. These splitting increases symbol duration by the number of subcarrier used and as a result of this phenomenon reducing of ISI due to multipath [4].

OFDM is adapted as the best transmission scheme for cognitive radio Network (CRN). The features and the ability 


\section{International Journal of Science and Research (IJSR) \\ ISSN (Online): 2319-7064 \\ Index Copernicus Value (2013): 6.14 | Impact Factor (2014): 5.611}

of OFDM system makes it fit for CR based transmission system. OFDM provides spectral efficiency, which is most require for CRN. This is because of subcarrier are very closely spaced and are overlapping without interference. Another advantage of OFDM is that it is very flexible and adaptive. The subcarrier can be turned on and off according to environment and such assist CR system dynamically. OFDM can be easily implement using Fast Fourier Transform (FFT), which can be done by digital signal processing using software [5].

There are two approaches for dynamic spectrum sensing i.e. Spectrum Underlay and Spectrum overlay. Spectrum overlay which is used to increase spectrum efficiency by granting secondary (i.e. unlicensed) user to opportunistically exploit unused frequency band of primary (i.e. licensed) user when the frequency band sensed as being unused in temporal and spatial domain. Spectrum underlay approach permits simultaneous sharing of all the frequency band available among the primary and secondary users while imposing severe restrictions on the transmission power of the secondary users. So as not to cause any harmful interference to active primary users [6].

The objective of this paper to write optimal algorithm to dynamically allocate the radio resources to the cognitive radio network which can maximize the throughput of the system within power and interference constraint provided by the alongside operating primary users and total power of CRN. In this paper, second part is related to particle swarm optimization, third part related to system model, fourth part related to simulation result, fifth part related to conclusion and last is references.

\section{Particle Swarm Optimization}

Now a day, for the optimization in the number of fields, particle swarm optimization (PSO) technique is used. PSO is one of the computational techniques in optimization field. PSO is nonlinear function of optimization and swarm intelligence based evolutionary algorithm. PSO is a biologically inspired algorithm motivated by social analogy. The main aim of PSO is to obtain global optimum of a real value function defined in a given space. It was inspired by the behavior of swarm to look for food. The PSO algorithms also make use of social behavior and movement dynamics of insects, birds and fishes. Let us take the example of fish for searching the food and searching behavior. The searching space of the fish can be considered as a search space and the fish in the shoal can be considered as a small particles denoting solution in the search space. The process of searching food is nothing but viewed as an optimization process. In this process, the members of the shoal compete among themselves and share an information with the partners to find the best solution of the problem.

The research has shown that when birds or fishes search for the food, they do it in groups (flocks and swarms) and not individually. The observation is based on the assumption that the information is shared inside the group among the individuals. The behavior of each individual is influenced by the behavior of the whole group. The PSO was develop through simulation of the simplified social system and has found robust in solving nonlinear optimization problems. The PSO algorithm help to produce simplified and good solution with lesser calculation, shorter time and stable convergence than any other conventional method [7].

PSO has two main component methodology i.e. Artificial life(A-life) in general, to bird flocking, fish schooling and swarming theory in particular. PSO is closely related to the Artificial Life and Evolutionary Algorithm. It uses a position velocity model in a swarm based searching process. A swarm consists of set of individuals or particles, each presenting a potential solution of problem being formulated. Each particle is characterized by its position and velocity in searching space. Due to because of position and velocity, it is helpful to searching exact space. In the similar way, the fitness value for each particle is evaluated by using the position and velocity to determine the solution performance using the avail or fitness function [8].

The position of a particle $i$ is denoted as $x i, k$ and its velocity by $v i, k, \mathrm{k}$ being iteration number. So the position and velocity can be denoted in vector form by $X i=[x i, 1$, $x i, 2 \ldots \ldots . . . x i, k]$ and $V i=[v i, 1, v i, 2 \ldots \ldots v i . k]$. An avail function (fitness function) is evaluate for a particle at each iteration using the position and velocity to find out of best solution, the solution for each particle is then stored in Pbest. After all iteration, the best solution for whole swarm is found out as Gbest.

The position and velocity updated as the following equations $x i, k+1=x i, k+v i, k+1$ (a)

$v i, k+1=\omega v i, k+c 1 r 1($ Pbest $-x i, k)+c 2 r 2($ Gbest $-x i, k)$ (b)

where, $\omega$ is an antenna weight. c1, c2 is a position accelerator, r1, r2 are random number.

The following steps in PSO Operation: -

(a) The position and velocity of particles are randomly initialized.

(b) The particles are evaluated by calculating their respective avail function. The current position and avail values are stored in best particle position called Pbest of each particle. And best position of whole swarm is stored in best position in group i.e. Gbest.

(c) Position and velocity of particles are updated according to equation (a) and (b). The particles are again evaluating by their avail value of the updated particle is greater than the current particle, the Pbest is replaced by updated position. Then, Gbest is updated after all iteration.

(d) When maximum number of iterations is over. Gbest is the optimal solution. Or else go to step (c) [9]. 


\section{System Model}

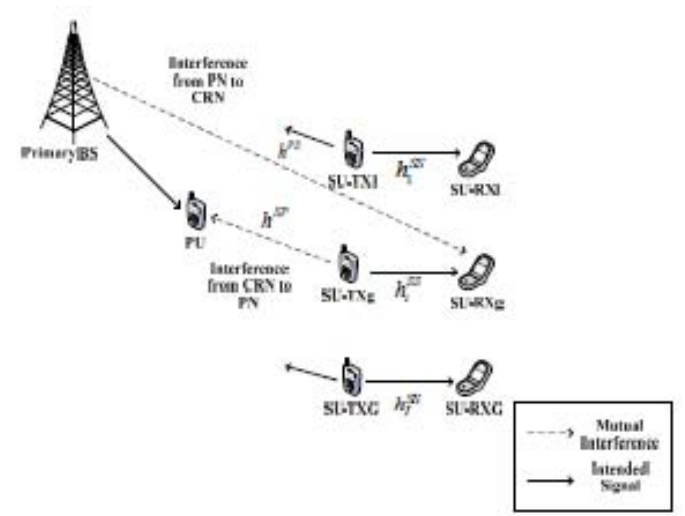

Figure 3.1: Cognitive Radio Network

Consider a Cognitive Radio Network Which consist of number of users that is multi-user. As illustrate in fig. 3.1. There is a primary base station (BS) that transmit the downlink traffic to the primary user(PU). The OFDM technique which is used in the communication of each secondary user(SU) and OFDM technique which is used to divides the available bandwidth into frequency-flat subchannels.

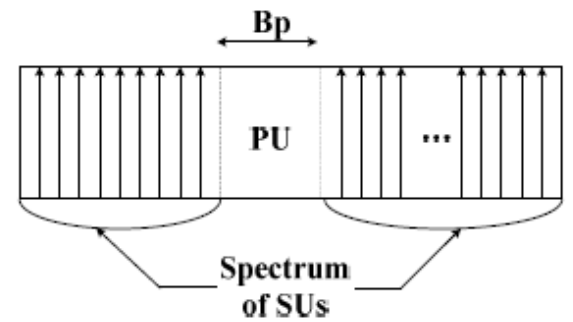

Figure 3.2: Spectrum of SU and PU in a CR system

In the fig. 3.2 let assume the spectrum of bandwidth Bp. And for the communication point of view, it may not require to transmit entire spectrum. So that SUs can exploit some part of the shared bandwidth, and each SU user has perfect knowledge of the aggregated interference from the PU. There are three kinds of channel gain in the network are used. These are $h^{\mathrm{ps}}, \mathrm{h}^{\mathrm{sp}}$ and $\mathrm{h}^{\mathrm{ss}}$ term which stand for channel gain from the primary BS to the SU receiver, the channel gain from the SU transmitter to the PU, and corresponding channel power gain on the link between SU transmitterreceiver pair respectively.

\section{Simulation Results}

In this paper, numerical simulation is used to evaluate the performance describe the power allocation problem for a CRN. In that, we initialize the users and power capacity of network. The simulation result shown as follows.

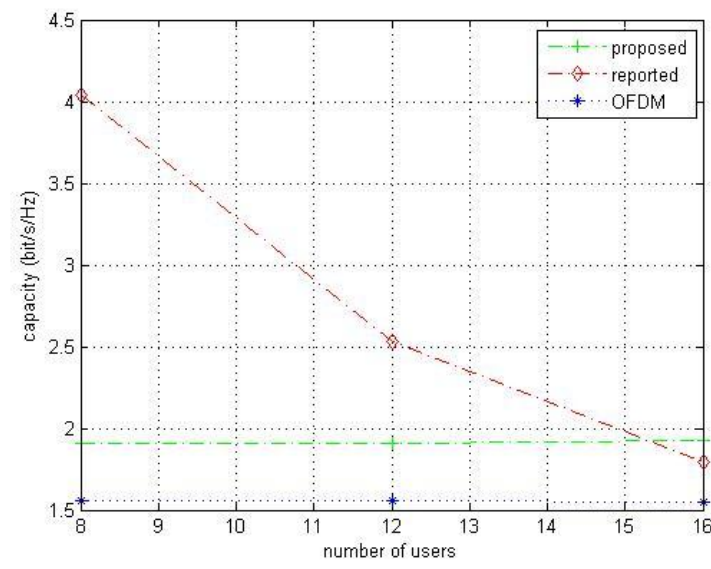

Figure 4.1: Comparison of Proposed and OFDM algorithm

In the above graph, it is shown as the variation of power capacity according to number of users which is related to the distributed algorithm and OFDM based algorithm for cognitive radio network. Capacity which is shown at y-axis and number of user on x-axis respectively. In the similar way, second simulation which is related the optimal value of power allocation.

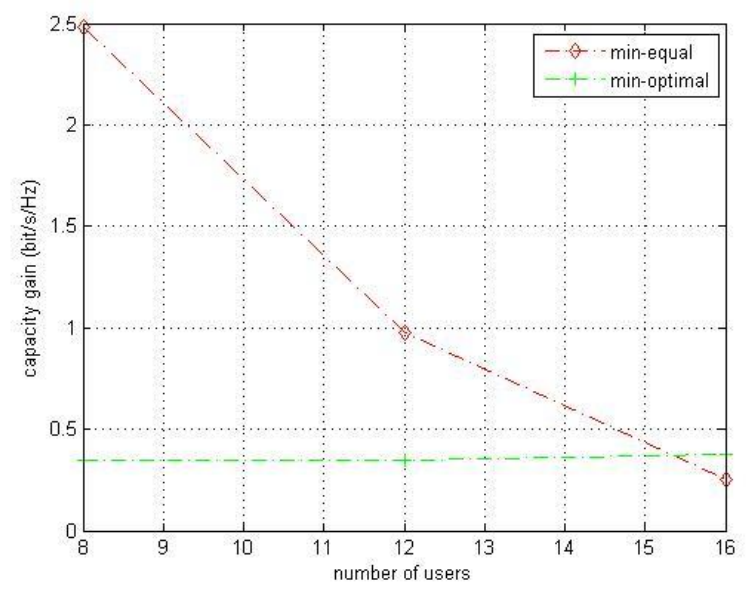

Figure 4.2: Optimal power allocation

In the above simulation, it is shown as the optimal value of capacity gain of power related to the number of users. Capacity gain on y-axis denoted as a $0.5,1,15,2,2.5$. and number of user as $8,9 \ldots, 16$. So the minimal optimum value of power which is shown in above figure.

\section{Conclusion}

The CR technology is used to increase the access of spectrum and improved communication service available to the public. This paper analyzed the power allocation problem, carried out a decentralized power allocation and maximize the throughput. The simulation result illustrated that proposed PSO algorithm. In the PSO algorithm, the optimal value of power allocation and maximum throughput are calculated. 


\section{References}

[1] Federal Communications commisions,"Notice of proposed rulemaking and order released,"Dec.30,2003.

[2] H. Mahmoud, T.Yucek, and H. Arslan,"OFDM for cognitive Radio: Merits And Challenges,"IEEE Wireless Commun.,vol.16, no. 2, pp. 6-15, Apr. 2009.

[3] C. C. Chai and Y. H. Chew,"Adaptive Joint Power and Bandwidth Control for spectrum sharing system," Proc IEEE VTC-2011 Spring yokohama, Japan, May 2013.

[4] S. Barbarossa, S. Sardellitti, and G. Scutari,"Jonit Optimization Of Detection Thresholds and Power Allocation in multiuser wideband cognitive Radio,"Proc COGIS, Paris, France,Nov.2009.

[5] T. Weiss, J. Hillenbrand, A. Krohn, and F.K.Jondral, "Mutual interference in OFDM-based spectrum pooling systems," Proc IEEE VTC 2004-Spring, vol. 4, pp. 1873-1877, Milan Italy ,May 2004.

[6] D. I. Kim, L. Le, and E. Hossain,"'Resourse allocation for cognitive Radios in Dynamic spectrum Accesss Environment,"Proc. Crowncom. 2008, pp. 1-6, Singapore, May 2008.

[7] C. Kloeck, D. Burgkhardt, F. K. Jondral, D. Grandblaise, S. Gault, and J.C. Dunat,"Bio-inspired algorithm for dynamic resourse allocation in cognitive wireless network," Proc. CrownCom. 2007, pp. 351356, Orlando, USA, Aug. 2007.

[8] R.C. Eberhert and J. Kennedy, "A new optimizer using particle swarm theory,"Proc. MHS 1995, pp. 39-43 ,Nagoya , Japan ,Oct 1995.

[9] R.C. Eberhert and J. Kennedy,"Particle swarm optimization,"Proc. IEEE ICNN 1995, pp.1942-1948, Perth, Australia, Nov.1995. 